\title{
Education in ultrasonography - a stringent and constant necessity
}

\author{
Radu Badea
}

Department of Ultrasonography, “Octavian Fodor" Institute of Gastroenterology and Hepatology, Iuliu Hatieganu University of Medicine and Pharmacy, Cluj-Napoca, Romania

Ultrasonography is the imaging method available to all clinical specialties. With a few exceptions, perhaps the psychiatric domain, there is no medical field that does not benefit from this method. Beginning with medical or surgical emergencies to the great clinical specialties and even the "smaller" ones - dermatology or physiotherapy, ultrasound examination is used to find complex or simple information required to eliminate diagnostic suspicion, to consolidate diagnosis, or differentiate between various diseases. Ultrasound practice may be global or partial, using the radio imaging model or the one that "extends" the clinical examination. The "point of care" concept is already well established. The equipment used is highly complex and has an exceptional diversity. The high level of miniaturization, portability and applications will optimize the image, whether conventional, elastographic, Doppler or CEUS are responsible for the efficiency of the technique. In the last years the concepts of telemedicine, artificial intelligence etc. have progressed rapidly.

The good practice aspect of the method has become increasingly complex. It is obvious that there is a need for a multimodal and multicriterial methodology. As the ultrasound image is generated by the examiner and its clinical relevance is, in most cases, the key to the examination, education in ultrasonography is more and more important. The key matter is a branching approach of ultrasonography, from an undergraduate level to that of a beginner or an advanced specialist.

Received Accepted

Med Ultrason

2018, Vol. 20, No 4, 411-412

Corresponding author: Prof. Radu Badea, MD, PhD

Department of Ultrasonography, "Octavian Fodor" Institute of Gastroenterology and Hepatology, Iuliu Hatieganu University of Medicine and Pharmacy 19-21 Croitorilor Street, 400162 Cluj-Napoca, Romania

E-mail: rbadea@umfcluj.ro
There are numerous expert groups of various domains of ultrasound, who are concerned with education [1]. There is already a consensus that education in ultrasonography can and should begin during medical school. A curriculum of ultrasonography from principles to clinical applications may be compulsory or optional. It could start in the first years of preclinical studies, as it is well known how enthusiastic students are regarding new technologies [2]. The offer of optional courses is welcome, given the students' interest for the image. And even more the "risk" that some of them would integrate just this one procedure. It is a risk that should be taken into account and investigated in depth [3]. However, medical students seem to assimilate and integrate rapidly the use of ultrasound in simple medical gestures such as palpation or organ measurement [4].

Logically, the next step is ultrasonography for the residents. Here too, we need a well-designed curriculum that would allow the resident to formulatean accurate diagnosis in his specialty and also in the related specialties. The specialist physician needs a curriculum that ensures continuous medical education and offers updated information to the practitioner. Finally, the doctors with long standing practice (the so called "seniors") may benefit from ultrasound education based on training of skills and abilities.

And not last, there is a need for continuous practice. Despite appearances, a simple primary training in ultrasonography is not sufficient to ensure a diagnosis of certainty. Every pathomorphological entity has a wide range of ultrasound aspects. The practitioner acquires information all the time, which is then processed, synthesized and made into a diagnosis. It is not enough to have high quality equipment. We must know how to use it in a constant manner.

It is our role, as experts, to contribute to the training in ultrasonography at various levels of complexity and over specific domains. It is the role of EFSUMB to iden- 
tify new ways to educate through centers of high excellence and expertise. Collaboration with medical schools is essential for this process. It is also the role of EFSUMB to ensure education in ultrasonography as a branch-like model! Only in this way will ultrasonography become a visual "stethoscope" and the fifth clinical movement of objective examination.

\section{References}

1. Mircea PA, Badea R, Fodor D, Buzoianu AD. Using ultrasonography as a teaching support tool in undergraduate medical education - time to reach a decision. Med Ultrason 2012;14:211-216.

2. Bell FE 3rd, Wilson LB, Hoppmann RA. Using ultrasound to teach medical students cardiac physiology. Adv Physiol Educ 2015;39:392-396.

3. Mouratev G, Howe D, Hoppmann R, et al. Teaching medical students ultrasound to measure liver size: comparison with experienced clinicians using physical examination alone. Teach Learn Med 2013;25:84-88.

4. Feilchenfeld Z, Dornan T, Whitehead C, Kuper A. Ultrasound in undergraduate medical education: a systematic and critical review. Med Educ 2017;51:366-378. 